\title{
PRIME MISURE SULLA RADIOATTIVITÀ DELLE PRECIPITAZIONI ATMOSFERICHE (*)
}

\author{
L. Santomauro - A. Gigna
}

Apparecchiatura strumentale. - I rilevamenti della radioattività furono compiuti con un contatore di Geiger-Muller sensibile alle radiazioni beta e gamma.

L'apparecchio consiste in un alimentatore ad $A T$ e in un amplificatore a $B F$ con alimentazione separata. Per l'alimentazione ad $A T$ ¿̀ stata adoperata una raddrizzatrice a due semionde, la $5 T 4$, collegata con le placche in parallelo.

Per il livellamento della corrente raddrizzata si sono impiegati due condensatori a carta da $0.25 \mu F-1500$ VL collegati in serie coil due resistenze da $5 M Q$ ciascuna, in parallelo, per assicurare una uniforme ripartizione del carico ai terminali dei condensatori di filtro. Pcr avere la regolazione della tensione negativa d'uscita una delle due resistenze è stata sdoppiata in un potenziametro da $2 M \stackrel{\text { e in }}{ }$ una fissa rla $3 M Q$.

Nell'amplificatore a $B F$ sono stati impiegati duc piccoli pentodi $R V 12 P 2000$ a grande amplificazione. L'apparecchio è munito di un altoparlante, di una presa per cuffia e di una uscita per numeratore o oscillografo.

Un diodo $V R 92$ provvede al raddrizzamento della corrente per l'alimentazione dell'amplificatore a $B F$. L'autotrasformatore dell'alimentatore è fornito di due secondari isolati tra loro per l'accensione delle valvole. Il gruppo di filtro è costituito come di consueto da due condensatori elettrolitici e da una piccola impedenza.

Il cilindro metallico del contatore $G \cdot M$ è stato collegato all' $A T$ negativa, l'elettrodo interno all'ingresso del circuito amplificatore mediante un cavo schermato e un piccolo condensatore. Particolare cura è stata posta nello schermare l'alimentatore ad $A T$.

Metodo delle misure. - Le misure vennero compiute dal febbraio 1951 fino al novembre 1952 con interruzione durante i mesi estivi.

Sono state fatte due serie di misure: la prima $\left(M^{\prime \prime}\right)$ con il contatore a vuoto e la seconda $\left(M^{\prime}\right)$ in presenza dell'acqua piovana.

(*) Comunicazione presentata al Convegno dell'Associazione Geofisica Italiana, tenuto a Roma il 17.18 giugno 1953. 
Come valore indicativo della radioattività delle precipitazioni è stata assunta la differenza $R$ delle medie degli impulsi delle due serie di misure. Queste ultime sono state eseguite su una quantità di acqua pari a 250 ce raccolta in una bacinella di vetro. Nel corso delle ricerche, la distanza bacinella-contatore è stata sempre la me-
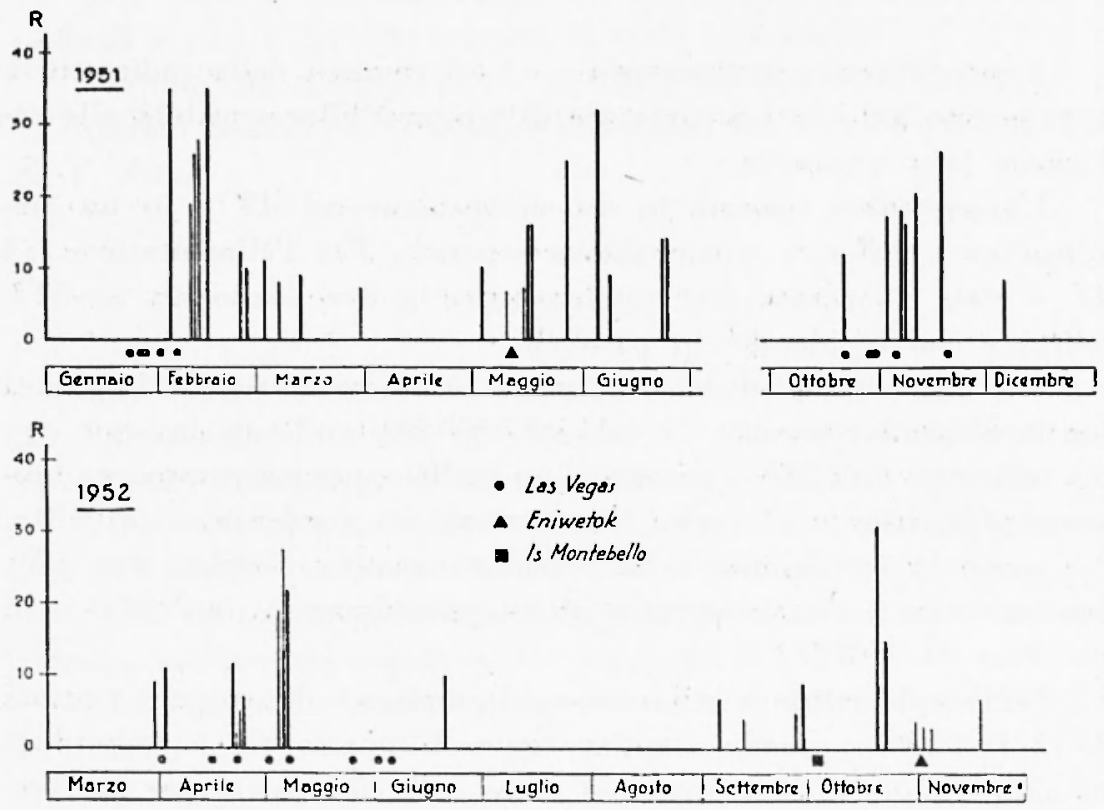

Fig. 1

dcsima. Allo scopo di ridurre l'entità delle radiazioni estranee, il sistema contatore-vaschetta è stato schermato mediante un riparo in piombo dello spessore di alcuni millimetri.

Per ciascuna esperienza è stato tenuto conto della pressione atmosferica, della tensione del contatore di $G-M$ nonché del tipo di precipitazione (pioggia a carattere temporalesco, pioggia normale, pioggia leggera, neve).

Le misure furono eseguite per la maggior parte a Milano, altre a Casalborgone (Torino) e a S. Cesarea Terme (Lecce).

La diversità nel numero degli impulsi $M^{\prime \prime}$ riscontrata nel corso delle esperienze sono dovute a variazioni ambientali e di tensione di rete Nelle future ricerche questo inconveniente sarà eliminato.

Per quanto riguarda le misurazioni dei giorni 31 ottobre, 2 e 4 novembre 1952, eseguite a $S$. Cesarea Terme, sulla costa del Mar Jonio, è da notare la particolare attendibilità dei valori ricavati in 


\begin{tabular}{|c|c|c|c|c|c|c|c|}
\hline N. & Data & Località & M" & $\mathbf{M}^{\prime}$ & $\mathbf{R}$ & $\begin{array}{l}\text { Durata } \\
\text { raccolta }\end{array}$ & $\begin{array}{c}\text { Tipo della } \\
\text { precipitazione }\end{array}$ \\
\hline 1 & $4-2-51$ & Milano & 70 & 105 & 35 & $0^{\mathrm{h}} 30^{\mathrm{m}}-3^{\mathrm{h}} 00^{\mathrm{m}}$ & Neve \\
\hline 2 & $10-2$ & $n$ & 81 & 100 & 19 & 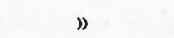 & Pioggia \\
\hline 3 & $11-2$ & ) & 79 & 105 & 26 & $n$ & Pioggia \\
\hline 4 & $12-2$ & 》) & 83 & 111 & 28 & 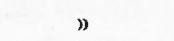 & Pioggia \\
\hline 5 & $15-2$ & ) & 80 & 115 & 35 & $n$ & Pioggia \\
\hline 6 & $24-2$ & 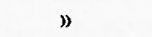 & 79 & 91 & 12 & ) & Neve \\
\hline 7 & $26 \cdot 2$ & $\eta$ & 80 & 90 & 10 & 》 & Pioggia forte \\
\hline 8 & 3.3 & y & 79 & 90 & 11 & $"$ & Pioggia leggera \\
\hline 9 & $7 \cdot 3$ & 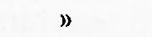 & 81 & 89 & 8 & ) & Pioggia \\
\hline 10 & $13-3$ & $》$ & 82 & 91 & 9 & $n$ & Pioggia \\
\hline 11 & 30.3 & $\eta$ & 88 & 95 & 7 & 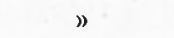 & Pioggia \\
\hline 12 & $3-5$ & ) & 89 & 99 & 10 & 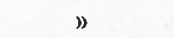 & Temporale \\
\hline 13 & $10-5$ & 》 & 89 & 96 & 7 & 》) & Temporale \\
\hline 14 & $15-5$ & $»$ & 82 & 89 & $i$ & $n$ & Temporale \\
\hline 15 & $16-5$ & 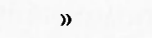 & 87 & 103 & 16 & ;" & Pioggia forte \\
\hline 16 & $17-5$ & $n$ & 88 & 104 & 16 & 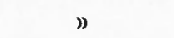 & Pioggia \\
\hline 17 & $27-5$ & ) & 88 & 113 & 25 & ” & Piogria \\
\hline 18 & $5 \cdot 6$ & " & 90 & 122 & 32 & " & Temporale \\
\hline 19 & $8-6$ & ) & 83 & 92 & 9 & $"$ & Temporale \\
\hline 20 & $23-6$ & $\eta$ & 93 & 107 & 14 & $"$ & Pioggia forte \\
\hline 21 & $21 \cdot 6$ & $\eta$ & 93 & $10 \bar{l}$ & $1+4$ & 》 & Pioggia \\
\hline 22 & $22-10$ & ) & 105 & 117 & 12 & 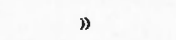 & Pioggia \\
\hline 23 & $3-11$ & ” & 77 & 94 & 17 & $\eta$ & Pioggia leggera \\
\hline 24 & 7.11 & 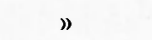 & 82 & 100 & 18 & 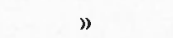 & Pioggia \\
\hline 25 & 8-11 & $\%$ & 96 & 112 & 16 & ” & Pioggia \\
\hline 26 & $11-11$ & $\eta$ & 98 & 118 & 20 & ) & Pioggia leggera \\
\hline 27 & $18-11$ & ) & 90 & 116 & 26 & 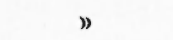 & Pioggia leggera \\
\hline 28 & $6-12$ & ) & 113 & 121 & 8 & ” & Pioggia leggera \\
\hline 29 & $30-3-52$ & 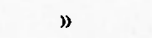 & 93 & 102 & 9 & $3^{\mathrm{h}} 30^{\mathrm{m}}$ & Pioggia \\
\hline 30 & 24 & $\eta$ & 103 & 114 & 11 & $6^{\mathrm{h}} 00^{\mathrm{m}}$ & Pioggia \\
\hline 31 & $21-4$ & $\eta$ & 116 & 128 & 12 & $0^{\mathrm{h}} 05^{\mathrm{m}}$ & Temporale \\
\hline 32 & $22-4$ & ) & 121 & 123 & 2 & $5^{\mathrm{h}} 15^{\mathrm{m}}$ & Pioggia leggera \\
\hline 33 & $23-1$ & 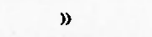 & 118 & 123 & 5 & $14^{\mathrm{h}} 30^{\mathrm{ml}}$ & Temp. e pioggia 1. \\
\hline 34 & $24-4$ & $\eta$ & 122 & 129 & 7 & $13^{\mathrm{h}} 30^{\mathrm{m}}$ & Pioggia \\
\hline 35 & $4-5$ & $\eta$ & 111 & 130 & 19 & $8^{\mathrm{h}} 00^{\mathrm{n}}$ & Pioggia \\
\hline 36 & $5-5$ & ) & 135 & 163 & 28 & $2^{\text {h }} 00 m$ & Pioggia \\
\hline 37 & $6-5$ & ) & 146 & 168 & 22 & $4^{h} 45^{\mathrm{m}}$ & Pioggia \\
\hline 38 & $19-6$ & $\eta$ & 123 & 133 & 10 & $2^{\mathrm{h}} 10^{\mathrm{nl}}$ & Temporale \\
\hline 39 & $5-9$ & Casalborg. & 72 & 79 & 7 & $0^{\mathrm{h}} 44^{\mathrm{m}}$ & Temporale \\
\hline 40 & 12.9 & $\eta$ & 66 & 70 & 4 & $0^{\mathrm{h}} 22^{\mathrm{m}}$ & Temporale \\
\hline 41 & $27-9$ & Milano & 111 & 116 & 5 & $0^{\mathrm{h}} 11^{\mathrm{m}}$ & Temporale \\
\hline 42 & 29.9 & " & 97 & 106 & 9 & $0^{\mathrm{lt}} 17^{\mathrm{nt}}$ & Pioggia \\
\hline 43 & $20-10$ & $\eta$ & 105 & 136 & 31 & $1^{\mathrm{h}} 00^{\mathrm{m}}$ & Pioggia legg. \\
\hline 44 & $22-10$ & 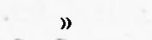 & 120 & 135 & 15 & $0^{\mathrm{hl}} 30^{\mathrm{m}}$ & Pioggia \\
\hline 45 & $31-10$ & S. Cesarea & 83 & 87 & 4 & $0^{\mathrm{l}} \quad 07 \mathrm{~m}$ & Pioggia \\
\hline 46 & $2-11$ & $m$ & 95 & 98 & 3 & $0^{\text {h }} 14^{m}$ & Temporale \\
\hline 47 & $4-11$ & $n$ & 98 & 101 & 3 & $0^{h} \quad 3^{m}$ & Pioggia forte \\
\hline 48 & $18-11$ & Milano & 120 & 127 & 7 & $3^{h} 49 m$ & Pioggia leggera \\
\hline
\end{tabular}


quanto risulta praticamente assente ogni possibile inquinamento dell'atmosfera dovuti a fumo o a pulviscolo.

11 numero $R$, clue nella tabella risulta dalla differenza $\left(M^{\prime}-M^{\prime \prime}\right)$ si riferisce a una scala arhitraria ed è il numero di impulsi al minuto registrati dal contatore. Tale numero indica, in media, una altivita pari a quella di acque termali non molto radioattive.

1 valori $M^{\prime}, M^{\prime \prime}, R$ nonché il tempo di raccolta delle precipitazioni stesse sono riportati nella tabella che precede.

Risultati. - Sull'ordinata di un sistema di coordinate normali si sono riportati i valori $R$ mentre sull'ascissa sono indicate le date delle esperienze (fiğ. 1).

A causa del carattere discontinuo del fenomeno considerato (precipitazioni atmosferichei è stata data la stessa rappresentazione usata

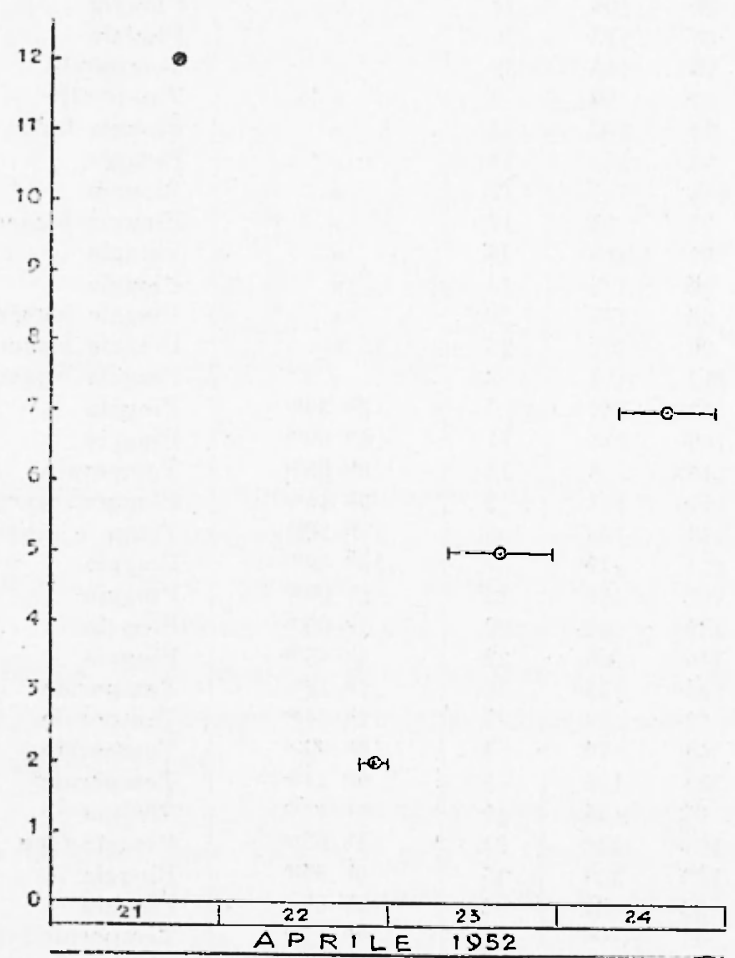

Fig. 2 da Alniba:, Pinoir, Pouradicr e Venet (1952).

$\mathrm{Si} \dot{e}$ constatato che si sono verificati degli aumenti temporanei della attività del. le precipitazioni nei periodi di tempo successivi ad esplosioni atomiche.

Le esplosioni atomiche prese in considerazione ai fini della presente ricerca e le cui date sono state desunte dai comunicati stampa, sono le seguenli : 24,27 e 28 gennaio, 1 e 6 febluraio 1951 a Las Vegas (U.S.A.). Il 14. maggio (data incer. ta) a Eniwetok (Oc. Pacifico). Il 22, 29, 30 otlobre, il 5 e 19 novembre 1951, il $1^{\circ}, 15$ e 22 aprile, il $1^{\circ}, 7$ e 25 maggio, il $1^{\circ}$ e il 7 giugno 1952 a Las Vegas (U.S.A.). Il 3 ottobre 1952 
alle Is. Montebello (Australia) e il $1^{\circ}$ novembre 1952 a Eniwetok (Oc. Pacifico).

Gli aumenti constatati in $R$ si sono verificati dopo circa 8 giorni dalle esplosioni di Las Vegas, dopo due settimane da quelle di Eniwetok e dopo circa tre settimane da quella delle Isole Montebello.

La correlazione tra la variazione del regime termico e della pressione atmosferica con l'aumento di $R$ ha dato risultato negativo. Così pure la maggiore attività nelle precipitazioni a carattere nevoso o temporalesco rispetto alle precipitazioni normali (A. Stefanizzi, 1950) a volte $\grave{e}$ in netto contrasto con gli aumenti di $R$ osservati.

Non è superfluo rammentare che la raclioattività naturale della pioggia è dovuta agli atomi radioattivi naturali ancorati all'aerosol atmosferico e non è improbabile che un aumento di particelle nellatmosfera possa maggiorare il numero dei nuclei di condensazione.

La misura della radioattività dell'alıa e media atmosfera per mezzo delle precipitazioni presenta il grave inconveniente di essere discontinua. Per contro, la misura della radioattività delle precipitazioni necessita di una tecnica più semplicistica di quella usata per l'attività dell'aria (Aliverti, 1933) e quelli sul valore della radioattivita delle precipitazioni (A. Stefanizzi, 1950), si ha:

$$
220 \times 10^{-18} \text { curie/ec (in media per l'aria) }
$$

da $10 \times$ a $51 \times 10^{-13}$ curie $/$ per le precipitazioni.

La radioattività media, quindi, di queste ultime è dell'ordine di $10^{4}$ volte l'attività dell'aria. Inoltre il grande volume d'aria scopato dalle precipitazioni stesse assicura un carattere più generale alla misura in quanto essa non si riferisce ad una zona limitata dell'atmosfera.

L'errore nelle misure dovuto alla radioattività delle precipitazioni, greneralmente non costante e legala invece al carattere della precipitazione stessa, viene mantenulo entro limiti abbastanza ristrelti, poiché essendo l'attività naturale rappresentata quasi esclusivamente da radiazioni alfa (A. Stefanizzi, 1950), essa non viene registrata dal nostro contatore, sensibile solo, come abbiamo grià accennato, a radiazioni più penetranti.

Va notato, infine, che il ripetersi per cinque volte del fenomeno dell'aumento temporaneo dell'attività delle precipitazioni qualche tempo dopo le esplosioni atomiche rende oltremodo improbabile la eventualità di una coincidenza casuale di fatti indipendenti tra loro e 
coincide, del resto, con i risultati conseguiti da alcuni ricercatori francesi (H. Garrigue, 1949, 1950, 1951 e 1952; Abribat e coll., 1952).

Può ora essere interessante considerare in un caso particolare (fig. 2) l'andamento della radioattività al verificarsi delle precipitazioni. Si sono scelti per questo i giorni $21,22,23$ e 24 aprile 1952 durante i quali a Milano piovve quasi senza interruzione e per i quali si hanno rispettivamente quattro misure. La radioattività misurata il giorno 21, all'inizio della pioggia, diede un valore $R=12$; il giorno seguente, subito dopo un violento acquazzone, la misura riferita alla susseguente pioggia leggera diede $R=2$; nei due giorni che seguirono, 23 e 24 aprile, la radioattività tornò ad aumentare gradatamente per riprendere il suo valore solito. La repentina diminuzione a 2 del valore di $R$ la si può attribuire al precedente acquazzone che avrebbe operato una intensa azione di scopatura dell'atmosfera riducendo così al minimo il valore della radioattività di quest'ultima.

Tra le 48 misure eseguite, ne sono state scelte 22 , che per la loro data c per $i$ valori presentati non sono state influenzate da esplosioni atomiche e si è potuto determinare il valore medio della radioattività normale delle precipitazioni attribuendo cosi un valore $R=9+1$.

Conclusione. - È da prospettare pertanto l'ipotesi che gli anormali aumenti dell'attività potrebbero essere dovuti appunto alle grandi quantità di prodotti radioattivi estremamente suddivisi che vengono sparsi per l'atmosfera in conseguenza delle esplosioni atomiche.

Da quanto sopra emerge l'importanza di codeste osservazioni e la necessità di continuarle con mezzi più completi e in maniera più sistematica.

Milano - Osservatorio Astronomico di Brera - Giugno 1953.

\section{RIASSUNTO}

Vengono esposti $i$ risultati ottenuti da misure di radioattività relativa delle precipitazioni atmosferiche in alcune località italiane (Casalborgone, Milano e S. Cesarea Terme), in seguito alle esplosioni atomiche. Tali misure sono state eseguite con un contatore di G-M, sensibile alle radiazioni beta e gamma. E stata trovata una maggiore attività nelle precipitazioni in seguito alle esplosioni atomiche. 


\section{SUMMARY}

During last years, from Februar 1951 to November 1952, the radioactivity of atmospheric precipitates uas tested with a G-M counter. This activity was no costant: indeed it became greater after atomic experiments at Las Vegas, Eniwetok and Montebello Is. Therefore these facts are probably associated. The activity increased about 8 days after Las Vegas experiments, two week after Enivetok experiments and about three weeks after Montebello Is. explosions. Gamma and beta rays of the precipitates have been measured with G-M counter and the bachground of cosmic radiation was considered in withdrawing the diagrams.

\section{BIBLIOGRAFIA}

(1) Abribat M, Pinoir R., Pouradien J, Venet A. M. (1952), Comptes Ren. n. 234, n. 11 .

(-) Aliverti G. (1931), L'Elettricista, Anno XXXX, n. 8, Roma.

(3) - (1931) Nuovo Cimento, VIII, n. 7.

(4) - (1932), Zeit. f. Geopliysik, J. 9; H. 1/2.

(5) - (1948), Rivista Geomineraria, Anno IX, n. 1.

(3) - e Lovera G. (1949), Annali di Geofisica, Vol. II, n. 1, 1949.

(7) Garaigue H. (1949), Comptes Rendus n. 228.

(8) - (1950), Comptes Rendus n. 230, n. 26.

(9) - (1951), Comptes Rendus n. 232, n. 10.

(10) - (1951), Comptes Rendus n. 233, n. 16.

(11) - (1951), Comptes Rendus n. 233, n. 23.

(15) - (1952), Comptes Rendus n. 235, n. 23.

(13) Jaufmann J. (1905), Met. Zeit., 22, 102.

(14) Priebsch J. A. (1932), Beitr. Geophysik, 36, n. 2/3.

(i5) Stefanizzi A. (1950), Journ. of Geof. Res, Vol. 55, n. 4.

(16) Werb (1949), Phys. Rev., Vol. 76, n. 3. 